\title{
THE SPLITTING OF CERTAIN SOLVABLE GROUPS'
}

\section{EUGENE SCHENKMAN}

Let $G$ be a finite group. We shall designate the commutator subgroup of $G$ by $G^{2}=[G, G]$; this is the group generated by all commutators $[g, h]=g h g^{-1} h^{-1}$. Inductively $G^{n}=\left[G^{n-1}, G\right]$ is defined to be the group generated by commutators of elements of $G$ with elements of $G^{n-1}$; and $G^{*}$ will designate $\bigcap_{n=1}^{\infty} G^{n}$. It should be recalled that $G$ is nilpotent if $G_{-}^{*}=E$, the subgroup consisting of the identity element, or equivalently, if $G$ is the direct product of $p$-groups.

Our object here is to show that when $G^{*}$ is Abelian then there is a nilpotent group $X$ so that $G=X G^{*}$ where $X \cap G^{*}=E$. If there are two such splittings of $G$ into $X G^{*}$ and $Y G^{*}$ then $Y$ and $X$ are conjugates by an element of $G^{*}$. If $x$ is in the center of $X$ then $x$ does not commute with any of its conjugates. As a consequence of the properties of the splitting it will follow that if $G$ has no center and $G^{*}$ is Abelian, then both $G$ and its group of automorphisms are contained in the holomorph of $G^{*}$.

We shall also give an example to show that the hypothesis that $G^{*}$ be nilpotent instead of Abelian is insufficient to insure a splitting of $G$ in this fashion.

The splitting of $G$. In order to show the existence of the splitting mentioned above we first prove the following fact.

Lemma. If $G / G^{*}$ is cyclic, that is, if $G$ is generated by $G^{*}$ and an element $x$, and if $G^{*}$ is Abelian, then every element of $G^{*}$ is of the form $[x, k]$ for some $k \in G^{*}$. Thus the map sending $k$ into $[x, k]$ is a 1-1 map of $G^{*}$ onto itself.

Proof. To prove this we shall use the following easily verified rules for commutators (cf. $[2$, p. 60]):

$$
\begin{aligned}
& {[a, b c]=[a, b][a, c]^{b} \quad \text { where } g^{b} \text { denotes } b g b^{-1},} \\
& {[a b, c]=[b, c]^{a}[a, c],}
\end{aligned}
$$

and

$$
[a, b]=[b, a]^{-1} \text {. }
$$

Presented to the Society, September 3, 1954; received by the editors July 21, 1954.

${ }^{1}$ This research was supported by the U. S. Air Force under contract number AF18(600)-790 monitored by the Office of Scientific Research. 
Then remembering that $G^{*}$ is an Abelian normal subgroup and that $G^{2}=G^{*}$ we have for $g, h$ in $G^{*}$

$$
[x, g h]=[x, g][x, h]=[x, g][x, h]
$$

and therefore also

$$
\left[x, g^{-1}\right]=[x, g]^{-1}=[g, x] \text { since } e=\left[x, g^{-1} g\right]=\left[x, g^{-1}\right][x, g] \text {. }
$$

Now the elements of $G$ are of the form $g x^{r}, h x^{s}$ for $r$ and $s$ integers and hence $\left[g x^{r}, h x^{s}\right]=\left[g x^{r}, h\right]\left[g x^{r}, x^{s}\right]=\left[x^{r}, h\right]\left[g, x^{s}\right]$. But $\left[x^{r}, h\right]$ $=\left[x^{r-1}, h\right] x[x, h]=\left[x^{r-1}, h^{x}\right][x, h]$ and therefore by an induction argument $\left[x^{r}, h\right]=[x, \bar{h}]$ for some $\bar{h}$ in $G^{*}$. Also $\left[g, x^{s}\right]=\left[x^{s}, g\right]^{-1}$ and therefore $\left[g, x^{s}\right]=[x, \bar{g}]$ for $\bar{g}$ in $G^{*}$. It follows that every commutator and hence in view of (1) every element of $G^{2}$ is of the form $[x, k]$ for some $k$ in $G^{*}$ as the lemma asserts. That the map sending $k$ into $[x, k]$ is a 1-1 map of $G^{*}$ onto itself follows readily from this.

CoRollary. If $H$ is a normal subgroup of $G$ contained in $G^{*}$ then $[x, H]=H$ where $[x, H]$ denotes the set of commutators $[x, h]$ for $h \in H$. If $K$ is the group generated by $x$ and $H$ then $K$ is not nilpotent and in fact $K^{*}=H$.

We can now prove the splitting theorem.

Theorem 1. If $G$ is a finite group so that $G^{*}$ is Abelian then $G$ contains a proper subgroup $X$ such that $G^{*} \cap X=E, G=G^{*} X$, and consequently $X$ is isomorphic to $G / G^{*}$ and is nilpotent.

Proof. $G^{*}$ is normal in $G$. We shall first consider the case where $G^{*}$ is minimal normal in $G$, that is $G^{*}$ does not properly contain any normal subgroup of $G$ other than $E$. Since $G$ is not nilpotent the $\Phi$ subgroup of $G$ (cf. $\left[2\right.$, p. 114]) does not contain $G^{2}$. Therefore there is a minimal set of generators of $G, g_{1}, \cdots, g_{k}$, where at least one of the generators, say $g_{k}$, is in $G^{2}$. Then $g_{1}, \cdots, g_{k-1}$ generate a proper subgroup $K$ of $G$. Since $G / G^{*}$ is nilpotent, $g_{1} G^{*}, \cdots, g_{k-1} G^{*}$ generate $G / G^{*}$ (cf. $\left[2\right.$, p. 114] again) and $G=G^{*} K$. Then $K \cap G^{*}$ is normal in $K$ and in $G^{*}$, hence in $G$. Since $K$ is a proper subgroup, $K \cap G^{*}$ must be $E$ and the theorem is proved when $G^{*}$ is minimal normal.

If $G^{*}$ is not minimal normal then we are going to show the existence of a subgroup $H$ properly contained in $G^{*}$ such that $[G, H]=H$. This is clearly true if $G^{*}$ has order not a power of a prime; hence suppose $G^{*}$ has order a power of a prime $p$. Since $G / G^{*}$ is a direct product of $p$-groups, $G$ has a normal non-nilpotent (cf. [1, pp. 98$102]$ ) subgroup $Q$ containing $G^{*}$ so that $Q / G^{*}$ has order a power of a prime $q \neq p$. Hence there is an element of $q$ power order not in the 
centralizer $Z$ of $G^{*}$. Since $Z$ is normal in $G$ and $G / Z$ is the direct product of $p$-groups, there is a central element of $G / Z$ of order a power of $q$ and consequently a normal subgroup $K$ of $G$ generated by $Z$ and an element $x$ of order a power of $q$. $x$ does not commute with all the elements of $G^{*}$; therefore $K$ is not nilpotent and we have $E \neq K^{*} \subset G^{*}, K^{*}$ normal in $G$. The elements of $K$ are of the form $x^{r} z$ for $r$ integral and $z$ in $Z$. Therefore if $g \in G^{*},\left[x^{r} z, g\right]=\left[x^{r}, g\right]$ and we see that if $L$ is the group generated by $x$ and $K^{*}$, then $L^{*}=K^{*}$. Now we can apply the corollary to the lemma to see that if $H$ is any normal subgroup contained in $K^{*}$ then $[x, H]=H$.

Now if $K^{*} \neq G^{*}$, then $K^{*}$ is the desired subgroup such that $\left[G, K^{*}\right]$ $=K^{*}$. If $K^{*}=G^{*}$ then any normal subgroup $H$ of $G$ contained in $G^{*}$ has the property that $[G, H]=H$ since $[x, H]=H$. In either event we can proceed by induction to finish the proof of the theorem. For let $H \neq E$ be properly contained in $G^{*}$ such that $[G, H]=H$. Then by an induction argument $G$ has a proper subgroup $K$ so that $G / H=K / H$ . $G^{*} / H$ or $G=K G^{*}$ with $K \cap G^{*} C H$. Then $[K, H]=H$ since $[G, H]$ $=H$ and $K^{*} \subset G^{*} \cap K \subset H$; hence $K^{*}=H \neq E$ and by the induction argument $K=X K^{*}$ where $X \cap K^{*}=E$. Finally $G=K G^{*}=X G^{*}$ and $X \cap G^{*} \subset K \cap G^{*} \subset H$; hence $X \cap G^{*} \subset H \cap X=K^{*} \cap X=E$ and the theorem is proved.

REMARK. We shall give here an example to show that the above type of splitting is in general impossible when $G^{*}$ is nilpotent even if $G / G^{*}$ is Abelian. For $p$ a prime not 2 let $H$ be a group of order $p^{4}$, generated by elements $a, b$, and $c$; $a$ and $b$ of order $p, c$ of order $p^{2}$, and $c^{p}=[a, b],[c, a]=[c, b]=e$, the identity. Let $h$ be an automorphism of $H$ sending $a$ into $a^{-1}, b$ into $b^{-1}$, and $c$ into $c$; and let $G$ be the holomorph of $H$ with $h$ of order $2 p^{4}$. Then $G^{*}$ consists of the group of order $p^{3}$ generated by $a$ and $b$. Since $c$ is of order $p^{2}$ and $c^{p}=[a, b]$ the impossibility of a splitting as in the theorem is clear.

On the conjugacy of the complements of $G^{*}$. If $G=A B$ where $A$ and $B$ are subgroups whose intersection is the identity we shall call $A$ a complement of $B$ in $G$. Our main result here is then the following.

Theorem 2. If $G^{*}$ is Abelian and if $X$ and $Y$ are two complements of $G^{*}$, then for some $h \in G^{*}, X=h Y h^{-1}$.

Proof. First suppose that $G^{*}$ is a minimal normal subgroup of $G$; then $G^{*}$ has order a power of some prime $p$. Let $x$ be of order $q$ prime to $p$ in the center of $X$. If $x$ is not in the centralizer of $G^{*}$ then $x$ and $G^{*}$ generate a normal subgroup $R$ of $G$ which is not nilpotent and therefore $R^{*}=G^{*}$ by the minimality condition on $G^{*}$. It follows from 
the corollary to the lemma of the last section that every element $h$ of $G^{*}$ is of the form $x g^{-1} g^{-1}$ for some $g$ in $G^{*}$.

Now there is a $y \neq e$ in $R \cap Y$ such that $y=x^{-1} h$ and therefore $y=g x^{-1} g^{-1}$. Suppose $Y \neq g X g^{-1}$; then since $G=G^{*}\left(g X g^{-1}\right)$ there is a $k$ in $Y$ so that $k=h g m g^{-1}$ for some $h$ in $G^{*}, h \neq e$, and $m$ in $X$. But then since $y$ is a conjugate of $x^{-1}, x$ in the center of $X$, it follows that $[k, y]$ $=\left[h g m g^{-1}, y\right]=[h, y]$; hence $[[k, y] \cdots y]=[[h, y] \cdots y] \neq e$ since $\left[y, G^{*}\right]=G^{*}$. But this is a contradiction of the nilpotency of $Y$ and we conclude that $Y=g X g^{-1}$ when $x$ is not in the centralizer of $G^{*}$.

If $x$ is in the centralizer of $G^{*}$ then $x$ is in the center of $G$ and since $x$ is in every Sylow $q$ group of $G, x$ is in $Y$. Then by an induction argument the theorem is true in $G /(x)$ and from this the theorem follows for $G$ when $G^{*}$ is minimal normal.

The general case now follows easily from this. As in the proof of Theorem 1 there is an $x$ and a normal subgroup $H$ of $G$ properly contained in $G^{*}$ such that $[x, H]=H$. Without loss in generality $x$ can be taken in $X$. By an induction argument, $Y / H=(g H) X / H(g H)^{-1}$ for some $g H$ in $G^{*} / H$ and hence if $g$ is an element of $g H$ then $g X g^{-1}$ is a complement of $K^{*}$ in the proper subgroup $K$ generated by $Y$ and $H$. But $[K, H]=H=K^{*}$ and by the induction assumption again there is an $h \in H$ so that $h\left(g X_{g^{-1}}\right) h^{-1}=Y$. This completes the proof of the theorem.

REMARK. If $h$ is in $G^{*}$ then either $h$ is in the center of $G$ or $h X h^{-1}$ $\neq X$. For if $h X h^{-1}=X$ then $X$ is normal in the group generated by $X$ and $h$; so also is $(h)$. Hence $[x, h] \subset X \cap(h)=E$ and $h$ is in the center of $G$.

REMARK. If $x$ is in the center of $X$ then $x$ does not commute with any of its conjugates. For if $y \neq x$ is a conjugate of $x$, then it is clear that $y=h x h^{-1}$ for some $h \in G^{*}$. If $x$ and $y$ commute, then $x$ commutes with $[h, x] \in G^{*}$ where $[h, x] \neq e$ since $y \neq x$. Hence $\left[x, G^{*}\right] \neq G^{*}$. Let $K$ be the group generated by $x$ and $G^{*}$; then $K^{*}$ is properly contained in $G^{*}$ and, being normal in $G, K$ contains a normal subgroup $H$ of $G$ so that either $[H, x]=H$ or $[H, x]=E$. Then by an induction argument assuming the statement true in $G / H$, we see that since $y=h x h^{-1}$ then $h$ must be in $H$ and consequently $[h, x]$ is in $H$. Since $[h, x] \neq e$ and commutes with $x$ it is not possible that $[H, x]=H$. On the other hand $[H, x]$ cannot be $E$ for then $[h, x]$ would be $e$. We thus get a contradiction by assuming that $x$ can commute with one of its conjugates.

On the group of automorphisms of $G$ when $G^{*}$ is Abelian and $G$ has no center. 
Theorem 3. If $G$ is a group with no center and $G^{*}$ is Abelian, then both $G$ and $A$, the group of automorphisms of $G$, are contained in the holomorph $R$ of $G^{*}$. Furthermore if $D$ and $F$ are complements of $G^{*}$ in $A$, then there is an $h \in G^{*}$ so that $h D h^{-1}=F$.

Proof. That $G$ is in $R$ follows from Theorem 1 ; for $G=G^{*} X$ where $G^{*}$ is normal in $G$ and $G \cap X=E$.

Now $G^{*}$ is a characteristic subgroup of $G$ and therefore every automorphism of $G$ maps $G^{*}$ into itself. Suppose $m$ is an automorphism of $G$ which commutes with all the elements of $G^{*}$. Let $L$ be the holomorph of $G$ and $m$ and let $Z$ be the centralizer of $G^{*}$ in $L$. Then $Z$ is normal in $L$ and hence for every $h \in G^{*},[m h, G] \subset Z \cap G=G^{*}$. In view of Theorem 2 there is an $h_{0}$ in $G^{*}$ so that $m h_{0}$ maps $X$ into itself; that is, $\left[m h_{0}, X\right] \subset X$. Therefore $\left[m h_{0}, X\right] \subset X \cap G^{*}=E$ and $m h_{0}$ commutes with every element of $X$ as well as of $G^{*}$; that is, $m h_{0}$ is the identity automorphism in $G$ or $m$ is the same as the inner automorphism determined by $h_{0}^{-1}$. It follows that $A / G^{*}$ is isomorphic to a subgroup $M$ of automorphisms of $G^{*}, M$ containing $X$ as a normal subgroup. Then $N$, the holomorph of $M$ with $G^{*}$ contains a copy of $G$ and the centralizer of this copy of $G$ is $E$. Then since $N$ and $A$ are both groups of automorphisms of $G$ having the same order, $A$ is isomorphic to $N$ and hence $A$ is a subgroup of $R$ as the theorem asserts.

We now show that if $D$ and $F$ are two complements of $G^{*}$ in $A$ then there is an $h \in G^{*}$ so that $F=h D h^{-1}$. It is clear that $D \cap G$ is normal in $D$ and is a complement of $G^{*}$ in $G$; hence there is an $h \in G^{*}$ so that $h(D \cap G) h^{-1}=F \cap G$. But $F \cap G$ determines $F$ completely; for if $F \cap G$ were normal in $F$ and also in $F^{\prime} \neq F$, then $F \cap G$ would be normal in the group generated by $F$ and $F^{\prime}$ which must necessarily intersect $G^{*}$ in a subgroup $Z \neq E$. But then $[Z, F \cap G]$ $C(F \cap G)$ and $[Z, F \cap G] \subset G^{*}$, whence it follows that $[Z, F \cap G]=E$ and $G$ has a nontrivial center contrary to hypothesis. It follows that $F \cap G$ determines $F$ completely, and then $h D h^{-1}$ must be $F$. This completes the proof of the theorem.

\section{BIBLIOGRAPHY}

1. H. Fitting, Beiträge zur Theorie der Gruppen endlicher Ordnung, Jber. Deutschen Math. Verein. vol. 48 (1938) pp. 77-141.

2. H. Zassenhaus, Theory of groups, translated, Chelsea, New York, 1949.

Louisiana State University and

The Institute for Advanced Study 